\title{
Phonon Decoherence of Quantum Dots in Photonic Structures: Broadening of the Zero-Phonon Line and the Role of Dimensionality
}

\author{
P. Tighineanu, ${ }^{1,2, *}$ C. L. Dreeßen, ${ }^{1}$ C. Flindt, ${ }^{3}$ P. Lodahl, ${ }^{1}$ and A. S. Sørensen ${ }^{1, \dagger}$ \\ ${ }^{1}$ Niels Bohr Institute, University of Copenhagen, Blegdamsvej 17, 2100 Copenhagen, Denmark \\ ${ }^{2}$ Max Planck Institute for the Science of Light, Staudtstraße 2, 91058 Erlangen, Germany \\ ${ }^{3}$ Department of Applied Physics, Aalto University, 00076 Aalto, Finland
}

(Received 15 February 2017; revised manuscript received 4 December 2017; published 21 June 2018)

\begin{abstract}
We develop a general microscopic theory describing the phonon decoherence of quantum dots and indistinguishability of the emitted photons in photonic structures. The coherence is found to depend fundamentally on the dimensionality of the structure resulting in vastly different performance for quantum dots embedded in a nanocavity (0D), waveguide (1D), slab (2D), or bulk medium (3D). In bulk, we find a striking temperature dependence of the dephasing rate scaling as $T^{11}$ implying that phonons are effectively "frozen out" for $T \lesssim 4 \mathrm{~K}$. The phonon density of states is strongly modified in 1D and 2D structures leading to a linear temperature scaling for the dephasing strength. The resulting impact on the photon indistinguishability can be important even at sub-Kelvin temperatures. Our findings provide a comprehensive understanding of the fundamental limits to photon indistinguishability in photonic structures.
\end{abstract}

DOI: 10.1103/PhysRevLett.120.257401

Disentangling a quantum system from its fluctuating environment is pivotal to the realization of coherent quantum bits. Controlling the sources of noise is particularly challenging in solid-state systems, which contain a myriad of mutually interacting quasiparticles. An example is semiconductor quantum dots (QDs), which have proven to be excellent quantum light sources [1]. Two important decoherence mechanisms of QDs are the fluctuating electrostatic [2,3] and spin [2,4,5] environments, but these can be neutralized under appropriate external control [6-9]. The electrostatic noise is particularly significant in engineered structures [10], but recent experiments have demonstrated how to efficiently suppress it [11,12]. The decoherence is then dominated by phonons, the acoustic vibrations of the crystal lattice [13-17]. Integrating the QDs into photonic devices is essential for obtaining deterministic and scalable light-matter interfaces [18-20]. Such photonic structures also contain a modified phononic environment due to the breakdown of translational symmetry. A unified description of how the modified phonon environment affects the coherence of QDs is lacking despite its vital importance for solid-state quantum optics [1]. Previous founding work concentrated on QDs in bulk media $[14,15]$, generic models for 1D and 3D phonon baths [21], or the special case of linear phonon coupling in nanowires [22] and carbon nanotubes [23].

Here we present a general microscopic theory describing the influence of phonons on the coherence of QDs and the indistinguishability of the emitted photons in photonic (nano)structures. The model is applied to the four experimentally relevant systems of an $\operatorname{In}(\mathrm{Ga}) \mathrm{As}$ QD in a cavity, waveguide, slab, or bulk medium corresponding to different geometric dimensionality from $0 D$ to $3 D$, see Fig. 1(d). The interaction with light is treated phenomenologically as a Markovian decay channel, which is the most abundant situation for applications such as coherent single-photon sources. The interplay between coherence and efficiency in the opposite limit of bulk phonons and non-Markovian light-matter interaction was recently explored in Ref. [24].

The impact of phonons on the photon emission from QDs can be seen clearly in the emission spectrum [13-17,22,25-35], which features broad sidebands superimposed on a narrow zero-phonon line (ZPL), cf. Fig. 1(a). The sidebands originate from rapid phonon emission or absorption on a picosecond time scale, see Fig. 1(c), while the ZPL arises from the long-time decay of coherence over nanosecond time scales. Because of the large spectral mismatch between the two processes, the incoherent sidebands can readily be filtered while maintaining a high efficiency of the photon source $[7,24,36]$. The fundamental limit to photon indistinguishability is therefore the interaction between the QD and phonons over long timescales, which is the main focus of the present Letter.

In a bulk medium, the broadening of the ZPL is described by an exciton-phonon coupling that is quadratic in phonon displacement [14], cf. Fig. 1(b). Here we obtain a simple expression for the dephasing rate, $\Gamma_{3 \mathrm{D}}$,

$\Gamma_{3 \mathrm{D}}=3 \pi \frac{v_{s}}{L} C_{Q}^{2} \int_{0}^{\infty} d(q L)(q L)^{10} e^{-(q L)^{2}} N_{q}\left(N_{q}+1\right)$,

where $C_{Q}$ is a dimensionless constant defined later, $L$ the radius of the QD wave function, $v_{s}$ the speed of sound, and 

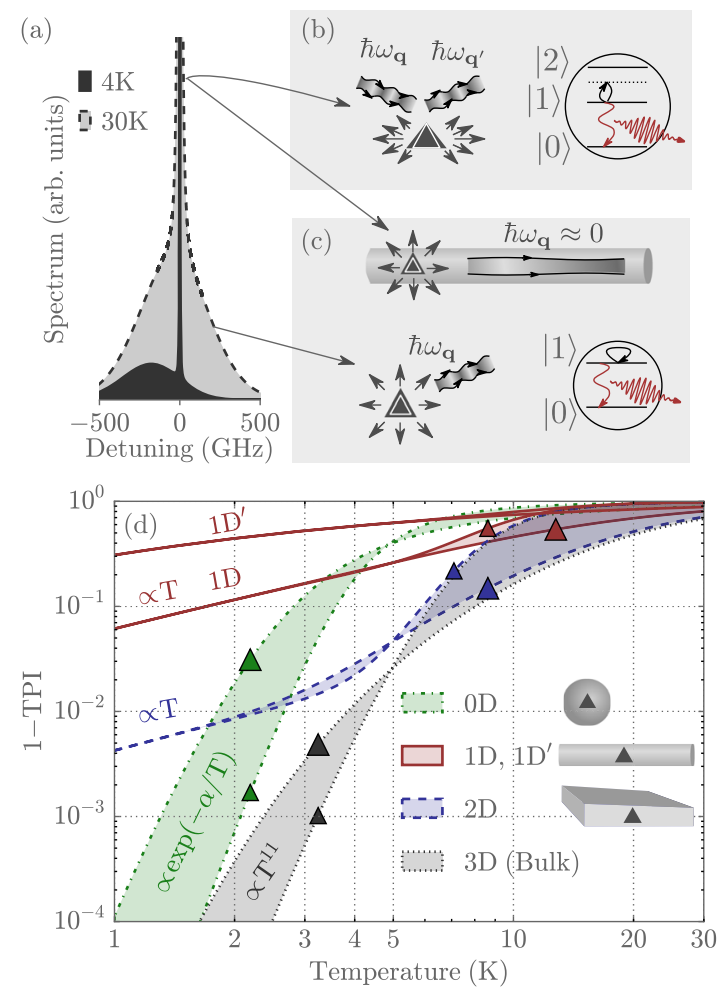

FIG. 1. Phonon dephasing of spontaneous emission from QDs. (a) The emission spectrum consists of a ZPL and broad sidebands. (b) The quadratic coupling represents scattering of phonons through virtual excitations to a higher state and leads to ZPL broadening. (c) The linear coupling is associated with the emission or absorption of phonons by the QD. In bulk this leads to phonon sidebands in the emission. In nanostructures, an additional mechanism broadens the ZPL through long-wavelength deformations. (d) Error in two-photon interference versus temperature for QDs embedded in structures with different dimensionality. 0D corresponds to a QD in the center of a sphere with radius $R=80 \mathrm{~nm}, 1 \mathrm{D}$ to a cylindrical waveguide with radius $\rho=$ $80 \mathrm{~nm}$ and the QD placed in the cross-sectional center (1D) or halfway off center $\left(1 \mathrm{D}^{\prime}\right), 2 \mathrm{D}$ to a QD in the center of a freestanding membrane with height $2 h=160 \mathrm{~nm}$, and $3 \mathrm{D}$ to a bulk medium. Each structure is represented by two curves that correspond to a large $(L=4.5 \mathrm{~nm})$ and small $(L=1.5 \mathrm{~nm})$ wave function denoted with large and small triangles, respectively.

$q$ and $N_{q}$ the phonon wave number and occupation number, respectively. Remarkably, when the thermal wavelength is larger than the QD size, $\lambda_{\mathrm{th}}>L$, corresponding to a temperature below a critical temperature $T_{c}=\hbar v_{s} / k_{B} L$, the phonons freeze leading to a rapid drop of the dephasing rate, cf. Fig. 1(d). This yields $\Gamma_{3 \mathrm{D}}\left(T<T_{c}\right) \simeq 3 \pi\left(v_{s} / L\right) \times$ $10 ! \times C_{Q}^{2}\left(T / T_{c}\right)^{11}$ leading to highly coherent processes at $T \lesssim 4 \mathrm{~K}$ for realistic QD sizes. Nanostructures on the other hand are finite and can thus expand freely, resulting in longwavelength vibrations that broaden the ZPL already within the linear exciton-phonon coupling; see Fig. 1(c). The latter competes with the quadratic coupling to yield a nontrivial temperature dependence of the photon indistinguishability; cf. Fig. 1(d). We find that these processes severely limit the coherence in $1 \mathrm{D}$ and $2 \mathrm{D}$ nanostructures.

To derive the results we generalize the formalism developed in Ref. [14] and consider arbitrary structures with the electron-phonon Hamiltonian

$$
H=\hbar \omega_{01}|1\rangle\left\langle 1\left|+\sum_{\mathbf{q}} \hbar \omega_{\mathbf{q}} a_{\mathbf{q}}^{\dagger} a_{\mathbf{q}}+V\right| 1\right\rangle\langle 1|,
$$

where $|1\rangle$ is the QD excited state, $a_{\mathbf{q}}^{\dagger}\left(a_{\mathbf{q}}\right)$ the creation (annihilation) operator for the phonon mode with momentum $\mathbf{q}$ and energy $\hbar \omega_{\mathbf{q}}$, and $\hbar \omega_{01}$ is the QD transition energy, cf. Fig. 1(b). We assume low temperatures such that the QD excited states are not populated. The interaction term, $V=V_{L}+V_{Q}$, comprises a linear and a quadratic term in phonon displacement

$$
\begin{aligned}
& V_{L}=\sum_{\mathbf{q}} L_{\mathbf{q}} A_{\mathbf{q}}, \quad V_{Q}=\sum_{b, m}\left(\sum_{\mathbf{q}} Q_{\mathbf{q} b}^{m} A_{\mathbf{q}}\right)^{2}, \\
& L_{\mathbf{q}}=M_{\mathbf{q} e}^{11}-M_{\mathbf{q} h}^{11}, \quad Q_{\mathbf{q} b}^{m}=\frac{M_{\mathbf{q} b}^{1 m}}{\sqrt{\Delta_{m}}},
\end{aligned}
$$

where $A_{\mathbf{q}}=a_{\mathbf{q}}+a_{\mathbf{q}}^{\dagger}, b=\{e, h\}$ denotes electron or hole, $M_{\mathbf{q} b}^{m n}$ is the electron-phonon matrix element, and $\Delta_{m}$ is the energy distance between the ground, $|1\rangle$, and $m$ th state of the QD with $m \geq 2$. Time-reversal symmetry implies that all quantities can be chosen real. The interaction with phonons is dominated by the deformation-potential coupling $[36,45]$

$$
M_{\mathbf{q} b}^{m n}=D_{b}\left\langle\psi_{b}^{m}\left|\nabla \cdot \mathbf{u}_{\mathbf{q}}\right| \psi_{b}^{n}\right\rangle,
$$

where $D_{b}$ is the deformation-potential constant, $\psi_{b}^{m}$ the wave function of the $m$ th state, and $\mathbf{u}_{\mathbf{q}}$ the phonon displacement.

After excitation at $t=0$, the QD coherence is described by the correlation function $P(t)=$

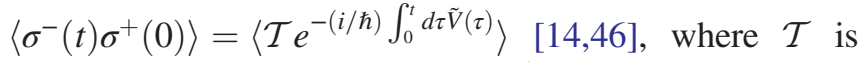
the time-ordering operator, and $\tilde{V}$ the potential in the interaction picture with respect to the free phonon Hamiltonian. $P(t)$ can be evaluated numerically exact using the cumulant expansion [14,47], but we follow a simplified approach that captures the essential physics. We find that the QD-phonon interaction is weak, such that retaining the first two terms in the cumulant expansion is sufficient. The distribution, therefore, becomes Gaussian and is completely specified by the mean and standard deviation of the noise $F(t)=-(i / \hbar) \int_{0}^{t} d \tau \tilde{V}(\tau)$. The truncation is thus equivalent to treating the phonon bath as a Gaussian noise source, which yields $P(t) \simeq$ $\exp \left(-i \mu_{F}\right) \exp \left[-\frac{1}{2}\left(\left\langle\mathcal{T} F^{2}(t)\right\rangle-\mu_{F}^{2}\right)\right]$, where $\mu_{F}=\langle F(t)\rangle$. In Ref. [36] we show that this provides an excellent 
approximation to the exact numerical result. Inserting Eq. (3) into the above expression yields

$$
\begin{aligned}
P(t) & =\exp \left[-i \mu_{F}+K_{L}(t)+K_{Q}(t)\right], \\
K_{L}(t) & =-\frac{i}{2 \hbar} \sum_{\mathbf{q}}\left|L_{\mathbf{q}}\right|^{2} \iint_{0}^{t} d t d t^{\prime} D_{\mathbf{q}}\left(t-t^{\prime}\right), \\
K_{Q}(t) & =\sum_{b m n} \iint_{0}^{t} d t d t^{\prime}\left[\sum_{\mathbf{q}} Q_{\mathbf{q} b}^{m} Q_{\mathbf{q} b}^{n} D_{\mathbf{q}}\left(t-t^{\prime}\right)\right]^{2},
\end{aligned}
$$

where $D_{\mathbf{q}}(t)=(-i / \hbar)\left[\left(N_{\mathbf{q}}+1\right) e^{-i \omega_{\mathbf{q}}|t|}+N_{\mathbf{q}} e^{i \omega_{\mathbf{q}}|t|}\right]$ is the phonon Green function. The function $K_{L}(t)$ stems from the linear electron-phonon interaction and is determined by a matrix element of the form $M_{\mathbf{q} b}^{11} \propto\left\langle\psi_{b}^{1}\left|\nabla \cdot \mathbf{u}_{\mathbf{q}}\right| \psi_{b}^{1}\right\rangle$, which shares the symmetry of the ground-state wave function, implying that $K_{L}(t)$ couples to symmetric acoustic deformations, cf. Fig. 1(c). On the other hand, $K_{Q}(t)$ is mediated by phonons that share the symmetry of the excited states.

In a bulk medium, the linear interaction $K_{L}(t)$ does not contribute to the long-time decay of coherence; see Fig. 2(a). The quadratic coherence function $P_{Q}(t)=$ $\exp \left[K_{Q}(t)\right]$ is evaluated for a spherical QD with Gaussian envelopes of radius $L$. Since $K_{Q}(t)$ is proportional to $\Delta_{m}^{-2}$, the inclusion of the first triply degenerate excited state, $m=n=2$, gives the dominant contribution. Evaluating $P_{Q}(t)$ numerically yields a Markovian decay over long time scales, cf. Fig. 2(a), with $\operatorname{Re}\left[K_{Q}(t)\right]=$ $-\Gamma_{3 \mathrm{D}} t$, and $\operatorname{Im}\left[K_{Q}(t)\right]$ contributes to a spectral shift. The dephasing rate can be calculated analytically by performing the time integration in Eq. (5) and using the long-time limit $\omega_{\mathbf{q}}^{-2} \sin ^{2} \omega_{\mathbf{q}} t \simeq \pi t \delta\left(\omega_{\mathbf{q}}\right)$. This leads to Eq. (1) with $C_{Q}=\left(D_{e}^{2} / \Delta_{e}+D_{h}^{2} / \Delta_{h}\right) / 3(2 \pi)^{2} \rho_{m} v_{s}^{2} L^{3}$. This is plotted in Fig. 2(b) for GaAs parameters: $v_{s}=4780 \mathrm{~ms}^{-1}$, mass density $\rho_{m}=5.37 \mathrm{~g} \mathrm{~cm}^{-3}, D_{e}=-14.6 \mathrm{eV}$, and $D_{h}=-4.8 \mathrm{eV}$. The energy distance to the excited states is taken to be $\Delta_{e}=2 \Delta_{h}=40 \mathrm{meV} \times L_{0} / L$ with
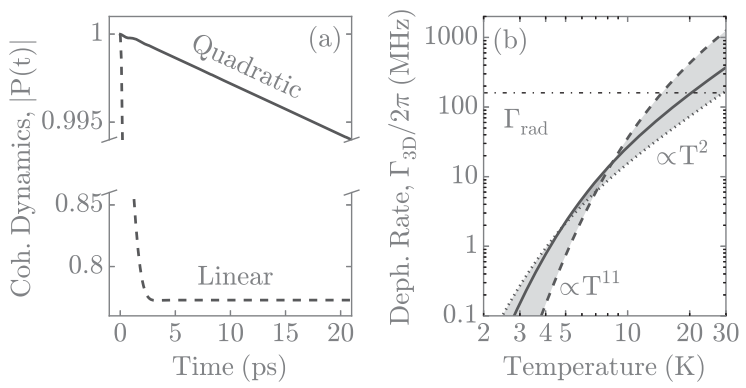

FIG. 2. Phonon dephasing in a bulk medium. (a) The linear (quadratic) exciton-phonon coupling affects the short-time (longtime) decay of coherence. Parameters: $T=10 \mathrm{~K}, L=3 \mathrm{~nm}$. (b) Phonon dephasing rate versus temperature for $L=1.5 \mathrm{~nm}$ (dashed line), $L=3 \mathrm{~nm}$ (solid line) and $L=4.5 \mathrm{~nm}$ (dotted line). The natural linewidth in a bulk medium is indicated by the dash-dotted line.
$L_{0}=3 \mathrm{~nm}$, in accordance with theoretical estimates and experimental results [48-54]. This choice of parameters is justified in Ref. [36].

To relate the phonon decoherence to the quality of the photons emitted by the QD, we study a Hong-Ou-Mandel setup [55]. Here, the second-order correlation function determines the two-photon indistinguishability (TPI), which ranges from 0 (no indistinguishability) to 1 (perfect indistinguishability). If (i) the QD-light interaction is Markovian, (ii) the excitation happens instantaneously, (iii) the QD is a perfectly antibunched source of single photons, and (iv) the noise is stationary, the TPI is [56]

$$
\mathrm{TPI}=\Gamma_{\mathrm{rad}} \int_{0}^{\infty} d \tau e^{-\Gamma_{\mathrm{rad}} \tau}|P(\tau)|^{2} .
$$

In bulk, $|P(t)| \simeq \exp \left(-\Gamma_{3 \mathrm{D}} t\right)$ leading to TPI $=\Gamma_{\text {rad }} /\left(\Gamma_{\text {rad }}+\right.$ $\left.2 \Gamma_{3 \mathrm{D}}\right)$ after filtering out the sidebands, where $\Gamma_{\mathrm{rad}} \simeq$ $2 \pi \times 160 \mathrm{MHz}$ is the radiative decay rate of the QD [57]. The resulting temperature dependence of the TPI is plotted in Fig. 1(d). Near-unity indistinguishabilities can be achieved at temperatures below a few Kelvin. Analytic solutions can also be found at high temperatures, $\Gamma_{3 \mathrm{D}}\left(T>T_{c}\right) \simeq 3 \pi^{3 / 2} \times(105 / 32)\left(v_{s} / L\right) \times C_{Q}^{2}\left(T / T_{c}\right)^{2}$, with a quadratic temperature dependence.

In the following we study phonon decoherence in nanostructures $[19,20,58,59]$. The short-time dynamics results in phonon sidebands that are shaped by the density of states, but this modification is not significant for the sizes considered here [36]. The long-time dynamics can be split into two contributions, $P_{\mathrm{ZPL}}(t)=P_{Q}(t) P_{\mathrm{L} 0}(t)$, where $P_{Q}(t)$ stems from the quadratic coupling, and $P_{\mathrm{L} 0}(t)$ is a nanostructure-specific low-frequency contribution to the linear coupling [21,22]. For simplicity we keep a fixed bulklike radiative decay rate throughout this work. In a realistic device this value may differ, in which case the results should be modified accordingly.

We start with a OD nanosphere cavity, which resembles the geometry of colloidal QDs embedded in spherical shells [60]. The long-time coherence is plotted in Fig. 3(a) and stems solely from the quadratic coupling $P_{Q}(t)$. The decay is strongly non-Markovian because the phonons are reflected at the boundary and interact with the QD periodically; see the inset of Fig. 3(a). A simple expression for $\operatorname{Re}\left[K_{Q}(t)\right]$ can be derived from Eq. (5) by using the long-time form $\sin ^{2}\left[\left(\omega_{j}-\omega_{j^{\prime}}\right) t\right] /\left(\omega_{j}-\omega_{j^{\prime}}\right)^{2} \simeq t^{2} \delta_{j j^{\prime}}$, where $j$ is the index of the confined acoustic mode. This results in $P_{\mathrm{ZPL}}(t)=\exp \left(-S^{2} t^{2}\right)$ with

$$
S^{2}=\frac{3}{2}\left(\frac{\pi}{2} \frac{v_{s}}{L} C_{Q}\right)^{2} \sum_{j} I_{j}^{4} \tilde{q}_{j}^{12} e^{-\tilde{q}_{j}^{2}} N_{\tilde{q}_{j}}\left(N_{\tilde{q}_{j}}+1\right),
$$

where $\tilde{q}_{j} \equiv q_{j} L$, and $I_{j}$ is a dimensionless normalization factor of the $(j, 1,0)$ spheroidal mode [36,61]. The resulting 

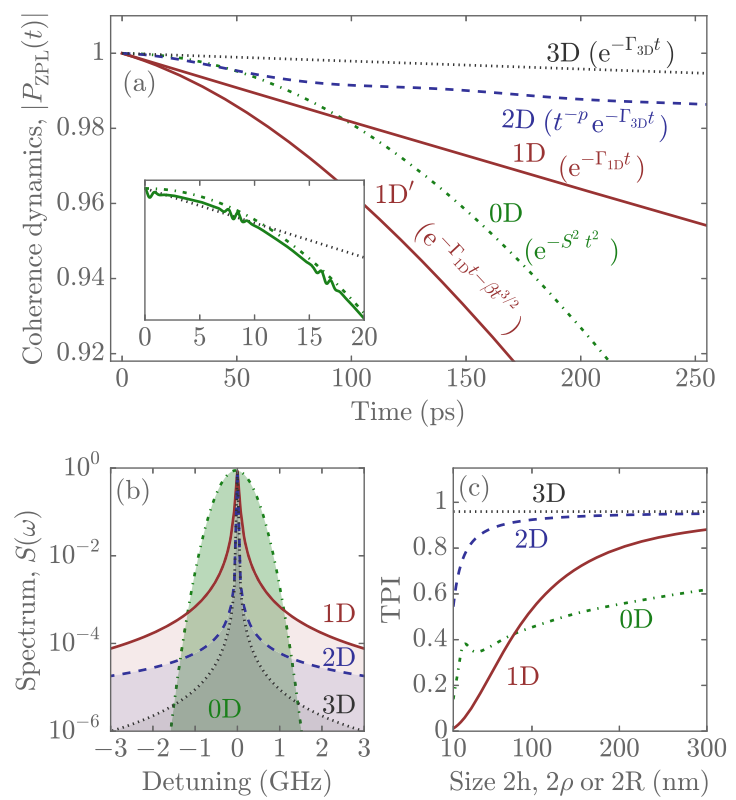

FIG. 3. Phonon dephasing in photonic structures and the role of dimensionality. (a) Decay of coherence for the same structures as in Fig. 1(d) except the 0D structure $(R=20 \mathrm{~nm})$. Inset: initial decay of coherence (0-20 ps) for bulk (dotted line), sphere (solid line), and the $t^{2}$ approximation for the sphere. (b) Corresponding emission spectrum. (c) Two-photon indistinguishability versus size of the structure. All plots are for $T=5 \mathrm{~K}, L=3 \mathrm{~nm}$.

emission spectrum without the radiative broadening, $S(\omega)=$ $\operatorname{Re} \int_{0}^{\infty} d t P_{\mathrm{ZPL}}(t) \exp (-i \omega t)$, is Gaussian as depicted in Fig. 3(b). The TPI yields $\mathrm{TPI}_{0 \mathrm{D}}=\sqrt{\pi} r_{s} \exp \left(r_{s}^{2}\right) \operatorname{erfc}\left(r_{s}\right)$, where $r_{s}=\Gamma_{\text {rad }} / 2 \sqrt{2} S$, and is plotted in Fig. 1(d). In general, the decoherence is stronger than in bulk. However, in the small temperature limit $\lambda_{\text {th }} \gg R$, the thermal energy is smaller than the lowest vibrational state of the sphere leading to negligible decoherence, $1-\mathrm{TPI}_{\mathrm{OD}} \propto$ $\exp \left(-\hbar \omega / 2 k_{B} T\right)$, as depicted in Fig. 1(d).

In the following, we discuss the dephasing of QDs embedded in 1D and 2D structures [20,62,63]. The quadratic interaction does not deviate significantly from bulk because $K_{Q}(t)$ is dominated by phonons with a wavelength comparable to the QD size while realistic photonic structures are much larger and do not affect those phonon modes. We therefore assume $K_{Q}^{1 \mathrm{D}} \simeq K_{Q}^{2 \mathrm{D}} \simeq-\Gamma_{3 \mathrm{D}} t$ [36]. This is different for the linear interaction $P_{\mathrm{L} 0}[22,23]$. For a freestanding 1D waveguide two families of acoustic modes contribute to dephasing with a finite $\nabla \cdot \mathbf{u}$ : longitudinal expansions of the rod with a linear dispersion and thus a constant density of states at $\omega \rightarrow 0$, and flexural modes that bend the rod with a quadratic dispersion and a diverging density of states [36]. The former yield a Markovian decay that was found in Ref. [22] for a cylinder but here is generalized to an arbitrary cross-sectional shape and QD position with the rate

$$
\Gamma_{\mathrm{L} 0}^{1 \mathrm{D}}=\frac{\left(D_{e}-D_{h}\right)^{2}(1-2 \nu)^{2} k_{B} T}{2 A \rho_{m} v_{1 \mathrm{D}}^{3} \hbar^{2}},
$$

where $A$ is the cross-sectional area, $\nu=0.299$ the GaAs Poisson ratio, and $v_{1 \mathrm{D}}=v_{s} \sqrt{3+2 \nu+2 /(\nu-1)}$ the phonon speed along the waveguide axis. The total decay, $\exp \left(-\Gamma_{1 \mathrm{D}} t\right)$, with $\Gamma_{1 \mathrm{D}}=\Gamma_{\mathrm{L} 0}^{1 \mathrm{D}}+\Gamma_{3 \mathrm{D}}$, is plotted in Fig. 3(a). The coupling to flexural modes, on the other hand, depends on the QD position, and ranges from no coupling at points of high symmetry (e.g., the center of a cylinder) to large coupling away from such points. In Fig. 3(a) we plot the numerically evaluated coherence decay of a QD placed off center at a distance $\rho / 2$ from the center of a cylindrical waveguide of radius $\rho\left(1 \mathrm{D}^{\prime}\right)$. The decay is non-Markovian, scaling as $P_{1 \mathrm{D}^{\prime}}=e^{-\Gamma_{1 \mathrm{D}} t-\beta t^{3 / 2}}$ [36]. In both cases, the error in TPI scales as $\propto T$ at low temperatures and is significant even for a waveguide with a diameter of hundreds of nanometers; see Figs. 1(d) and 3(c).

Next we consider a QD embedded in a freestanding 2D membrane with thickness $2 h$. To evaluate the coherence, we approximate the dispersion of the fundamental vibrational mode [64] as $\omega=v_{2 \mathrm{D}} q_{\|}$and discard the modes with $q_{\|}>h^{-1}$ [36], where $v_{2 \mathrm{D}}=v_{s} \sqrt{1-2 \nu} /(1-\nu)$, and $q_{\|}$is the in-plane wave number. The linear scaling of the density of states with $\omega$ yields

$$
\operatorname{Re}\left[K_{2 \mathrm{D}}\right]=-p\left[\gamma_{E}+\int_{\tilde{t}}^{\infty} d \tau \frac{\cos \tau}{\tau}+\ln \tilde{t}\right]-\Gamma_{3 \mathrm{D}} t
$$

Here, $p=\left(D_{e}-D_{h}\right)^{2}(1-2 \nu)^{2} k_{B} T / 4 \pi \rho_{m} h v_{2 \mathrm{D}}^{4}(1-\nu)^{2} \hbar^{2}$, $\tilde{t}=v_{2 \mathrm{D}} t / h$, and $\gamma_{E}$ is the Euler-Mascheroni constant. The coherence is plotted in Fig. 3(a) and results in a TPI that is dominated by the linear interaction at low temperatures as shown in Fig. 1(d). At long times $\left(t \gg h / v_{2 \mathrm{D}}\right)$, Eq. (9) can be simplified to $P_{2 \mathrm{D}}=\left(v_{2 \mathrm{D}} t / h\right)^{-p} \exp \left(-\Gamma_{3 \mathrm{D}} t\right)$. QDs positioned away from the membrane center would also couple to flexural modes with quadratic dispersion resulting in a

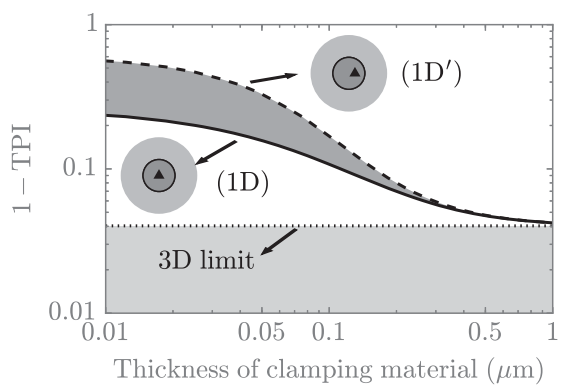

FIG. 4. Suppressing phonon dephasing by clamping the photonic structures. The plot shows the photon infidelity versus thickness of $\mathrm{SiO}_{2}$ (gray) surrounding a GaAs waveguide (dark gray) of radius $80 \mathrm{~nm}$ with a QD (small black triangle) in the center (solid line) and halfway off center (dashed line). Parameters: $T=5 \mathrm{~K}, L=3 \mathrm{~nm}$, $v_{s, \mathrm{SiO} 2}=5848 \mathrm{~ms}^{-1}, \rho_{m, \mathrm{SiO} 2}=2.2 \mathrm{~g} \mathrm{~cm}^{-3}, \nu_{\mathrm{SiO} 2}=0.17$. 
Markovian dephasing. Contrary to these examples, the 0D structure has a vanishing density of states at low frequencies. As used above, the dephasing is therefore only due to the quadratic coupling.

The theory developed above directly points towards methods of suppressing the malign impact of phonons. By mechanically clamping the structure, the coupling to the fundamental vibrational mode can be suppressed. This may be achieved by immersing the freestanding structure into another material (e.g., glass or semiconductor [65]) with a lower refractive index such that the light is still guided. We find that thicknesses as small as $1 \mu \mathrm{m}$ are sufficient to fully suppress the decoherence; see Fig. 4. This may provide a viable approach to obtain near-unity TPI.

In conclusion, we find that the degree of confinement of the nanostructure has a significant impact on the coherence. Bulk (3D) and maximally confined (0D) structures dephase the ZPL solely due to the quadratic exciton-phonon coupling, which becomes negligible at low temperatures, specifically for $\lambda_{\mathrm{th}} \gg L$ and $\lambda_{\mathrm{th}} \gg R$, respectively. The decoherence in 1D and 2D structures is enhanced by long-wavelength vibrations mediated by the linear exciton-phonon coupling and can be important even at subKelvin temperatures. The situation is more involved in the case of more complex structures such as photonic-crystal devices. We expect a photonic-crystal membrane to exhibit worse coherence than a 2D membrane of same thickness due to the holes that would slow down the long-wavelength phonons; see Eq. (9). A detailed calculation of the decoherence in photonic-crystal structures is an interesting question for further investigation.

We thank Johan R. Ott and Anna Grodecka-Grad for their collaboration at an early stage of this work, and Jake Iles-Smith and Jesper Mørk for useful discussions. We gratefully acknowledge the financial support from the Danish Council for Independent Research, the European Research Council (ERC consolidator grant "QIOS" and advanced grant "SCALE"), and the Academy of Finland through its Centre of Excellence program (Project No. 312299).

Note added.-Recently, a related preprint appeared (and was recently published [66]), which studies the phonon decoherence in bulk systems.

*petru.tighineanu@mpl.mpg.de †anders.sorensen@nbi.ku.dk

[1] P. Lodahl, S. Mahmoodian, and S. Stobbe, Interfacing single photons and single quantum dots with photonic nanostructures, Rev. Mod. Phys. 87, 347 (2015).

[2] A. V. Kuhlmann, J. Houel, A. Ludwig, L. Greuter, D. Reuter, A. D. Wieck, M. Poggio, and R. J. Warburton, Charge noise and spin noise in a semiconductor quantum device, Nat. Phys. 9, 570 (2013).
[3] C. Arnold, V. Loo, A. Lemaitre, I. Sagnes, O. Krebs, P. Voisin, P. Senellart, and L. Lanco, Cavity-Enhanced RealTime Monitoring of Single-Charge Jumps at the Microsecond Time Scale, Phys. Rev. X 4, 021004 (2014).

[4] B. Urbaszek, X. Marie, T. Amand, O. Krebs, P. Voisin, P. Maletinsky, A. Högele, and A. Imamoglu, Nuclear spin physics in quantum dots: An optical investigation, Rev. Mod. Phys. 85, 79 (2013).

[5] A. Delteil, W. B. Gao, P. Fallahi, J. Miguel-Sanchez, and A. Imamoğlu, Observation of Quantum Jumps of a Single Quantum Dot Spin Using Submicrosecond Single-Shot Optical Readout, Phys. Rev. Lett. 112, 116802 (2014).

[6] A. V. Kuhlmann, J. H. Prechtel, J. Houel, A. Ludwig, D. Reuter, A. D. Wieck, and R. J. Warburton et al., Transformlimited single photons from a single quantum dot, Nat. Commun. 6, 8204 (2015).

[7] N. Somaschi et al., Near-optimal single-photon sources in the solid state, Nat. Photonics 10, 340 (2016).

[8] X. Ding, Y. He, Z.-C. Duan, N. Gregersen, M.-C. Chen, S. Unsleber, S. Maier, C. Schneider, M. Kamp, S. Höfling, C.-Y. Lu, and J.-W. Pan, On-Demand Single Photons with High Extraction Efficiency and Near-Unity Indistinguishability from a Resonantly Driven Quantum Dot in a Micropillar, Phys. Rev. Lett. 116, 020401 (2016).

[9] Y.-M. He, Y. He, Y.-J. Wei, D. Wu, M. Atatüre, C. Schneider, S. Höfling, M. Kamp, C.-Y. Lu, and J.-W. Pan, On-demand semiconductor single-photon source with near-unity indistinguishability, Nat. Nanotechnol. 8, 213 (2013).

[10] A. Majumdar, E. D. Kim, and J. Vučković, Effect of photogenerated carriers on the spectral diffusion of a quantum dot coupled to a photonic crystal cavity, Phys. Rev. B 84, 195304 (2011).

[11] G. Kiršanskè et al., Indistinguishable and efficient single photons from a quantum dot in a planar nanobeam waveguide, Phys. Rev. B 96, 165306 (2017).

[12] H. Thyrrestrup et al., Quantum optics with near-lifetimelimited quantum-dot transitions in a nanophotonic waveguide, Nano Lett. 18, 1801 (2018).

[13] L. Besombes, K. Kheng, L. Marsal, and H. Mariette, Acoustic phonon broadening mechanism in single quantum dot emission, Phys. Rev. B 63, 155307 (2001).

[14] E. A. Muljarov and R. Zimmermann, Dephasing in Quantum Dots: Quadratic Coupling to Acoustic Phonons, Phys. Rev. Lett. 93, 237401 (2004).

[15] T. Grange, Decoherence in quantum dots due to real and virtual transitions: A nonperturbative calculation, Phys. Rev. B 80, 245310 (2009).

[16] A. Thoma et al., Exploring Dephasing of a Solid-State Quantum Emitter via Time- and Temperature-Dependent Hong-Ou-Mandel Experiments, Phys. Rev. Lett. 116, 033601 (2016).

[17] J. M. Zajac and S. I. Erlingsson, Temperature dependency of resonance fluorescence from InAs/GaAs quantum dots: Dephasing mechanisms, Phys. Rev. B 94, 035432 (2016).

[18] J. Claudon et al., A highly efficient single-photon source based on a quantum dot in a photonic nanowire, Nat. Photonics 4, 174 (2010).

[19] O. Gazzano, S. Michaelis de Vasconcellos, C. Arnold, A. Nowak, E. Galopin, I. Sagnes, L. Lanco, A. Lemaître, and 
P. Senellart, Bright solid-state sources of indistinguishable single photons, Nat. Commun. 4, 1425 (2013).

[20] M. Arcari et al., Near-Unity Coupling Efficiency of a Quantum Emitter to a Photonic Crystal Waveguide, Phys. Rev. Lett. 113, 093603 (2014).

[21] G. Palma, K.-A. Suominen, and A. K. Ekert, Quantum computers and dissipation, Proc. R. Soc. A 452, 567 (1996).

[22] G. Lindwall, A. Wacker, C. Weber, and A. Knorr, ZeroPhonon Linewidth and Phonon Satellites in the Optical Absorption of Nanowire-Based Quantum Dots, Phys. Rev. Lett. 99, 087401 (2007).

[23] C. Galland, A. Högele, H. E. Türeci, and A. Imamoğlu, Non-Markovian Decoherence of Localized Nanotube Excitons by Acoustic Phonons, Phys. Rev. Lett. 101, 067402 (2008).

[24] J. Iles-Smith, D. P. S. McCutcheon, A. Nazir, and J. Mørk, Phonon limit to simultaneous near-unity efficiency and indistinguishability in semiconductor single photon sources, Nat. Photonics 11, 521 (2017).

[25] E. A. Muljarov, T. Takagahara, and R. Zimmermann, Phonon-Induced Exciton Dephasing in Quantum Dot Molecules, Phys. Rev. Lett. 95, 177405 (2005).

[26] P. Kaer, T. R. Nielsen, P. Lodahl, A. P. Jauho, and J. Mørk, Microscopic theory of phonon-induced effects on semiconductor quantum dot decay dynamics in cavity QED, Phys. Rev. B 86, 085302 (2012).

[27] K. H. Madsen, P. Kaer, A. Kreiner-Moller, S. Stobbe, A. Nysteen, J. Mork, and P. Lodahl, Measuring the effective phonon density of states of a quantum dot in cavity quantum electrodynamics, Phys. Rev. B 88, 045316 (2013).

[28] A. Nazir and D. P.S. McCutcheon, Modelling excitonphonon interactions in optically driven quantum dots, J. Phys. Condens. Matter 28, 103002 (2016).

[29] P. Borri, W. Langbein, U. Woggon, V. Stavarache, D. Reuter, and A.D. Wieck, Exciton dephasing via phonon interactions in InAs quantum dots: Dependence on quantum confinement, Phys. Rev. B 71, 115328 (2005).

[30] M. Bayer and A. Forchel, Temperature dependence of the exciton homogeneous linewidth in $\mathrm{In}_{0.60} \mathrm{Ga}_{0.40} \mathrm{As} / \mathrm{GaAs}$ self-assembled quantum dots, Phys. Rev. B 65, 041308 (2002).

[31] W. Langbein, P. Borri, U. Woggon, V. Stavarache, D. Reuter, and A.D. Wieck, Radiatively limited dephasing in InAs quantum dots, Phys. Rev. B 70, 033301 (2004).

[32] K. Roy-Choudhury, N. Mann, R. Manson, and S. Hughes, Resonance fluorescence spectra from coherently driven quantum dots coupled to slow-light photonic crystal waveguides, Phys. Rev. B 93, 245421 (2016).

[33] K. Roy-Choudhury and S. Hughes, Quantum theory of the emission spectrum from quantum dots coupled to structured photonic reservoirs and acoustic phonons, Phys. Rev. B 92, 205406 (2015).

[34] P. Kaer, P. Lodahl, A. P. Jauho, and J. Mork, Microscopic theory of indistinguishable single-photon emission from a quantum dot coupled to a cavity: The role of non-Markovian phonon-induced decoherence, Phys. Rev. B 87, 081308 (2013).

[35] S. Rudin, T.L. Reinecke, and M. Bayer, Temperature dependence of optical linewidth in single InAs quantum dots, Phys. Rev. B 74, 161305 (2006).
[36] See Supplemental Material at http://link.aps.org/ supplemental/10.1103/PhysRevLett.120.257401, for a detailed description of the theory and justification of the used approximations, which includes Refs. [37-44].

[37] P. W. Fry et al., Inverted Electron-Hole Alignment in InAsGaAs Self-Assembled Quantum Dots, Phys. Rev. Lett. 84, 733 (2000).

[38] J. L. Skinner and D. Hsu, Pure dephasing of a two-level system, J. Phys. Chem. 90, 4931 (1986).

[39] P. Tighineanu, A. S. Sørensen, S. Stobbe, and P. Lodahl, Unraveling the Mesoscopic Character of Quantum Dots in Nanophotonics, Phys. Rev. Lett. 114, 247401 (2015).

[40] I. Vurgaftman, J. R. Meyer, and L. R. Ram-Mohan, Band parameters for iii-v compound semiconductors and their alloys, J. Appl. Phys. 89, 5815 (2001).

[41] B. A. Auld, Acoustic Fields and Waves in Solids (John Wiley \& Sons, New York, 1973).

[42] M. A. Stroscio, K. W. Kim, S. Yu, and A. Ballato, Quantized acoustic phonon modes in quantum wires and quantum dots, J. Appl. Phys. 76, 4670 (1994).

[43] P. Tighineanu, R. Daveau, E. H. Lee, J. D. Song, S. Stobbe, and P. Lodahl, Decay dynamics and exciton localization in large GaAs quantum dots grown by droplet epitaxy, Phys. Rev. B 88, 155320 (2013).

[44] L. Landau and E. Lifshitz, Theory of Elasticity, 2nd ed. (Pergamon Press, New York, 1970).

[45] T. Takagahara, Theory of exciton dephasing in semiconductor quantum dots, Phys. Rev. B 60, 2638 (1999).

[46] G. D. Mahan, Many-Particle Physics (Springer Science \& Business Media, New York, 2013).

[47] R. Kubo, Generalized cumulant expansion method, J. Phys. Soc. Jpn. 17, 1100 (1962).

[48] I. Schwartz et al., Deterministic generation of a cluster state of entangled photons, Science 354434 (2016).

[49] C. Pryor, Eight-band calculations of strained InAs/GaAs quantum dots compared with one-, four-, and six-band approximations, Phys. Rev. B 57, 7190 (1998).

[50] K. Schmidt, G. Medeiros-Ribeiro, M. Oestreich, and P. M. Petroff, Excited states in InAs self-assembled quantum dots, Photonics West (International Society for Optics and Photonics, Bellingham WA, 1996), Vol. 185.

[51] S. Noda, T. Abe, and M. Tamura, Mode assignment of excited states in self-assembled InAs/GaAs quantum dots, Phys. Rev. B 58, 7181 (1998).

[52] M. Grundmann, O. Stier, and D. Bimberg, InAs/GaAs pyramidal quantum dots: Strain distribution, optical phonons, and electronic structure, Phys. Rev. B 52, 11969 (1995).

[53] L. Landin, M. Miller, M.-E. Pistol, C. Pryor, and L. Samuelson, Optical studies of individual InAs quantum dots in GaAs: few-particle effects, Science 280, 262 (1998).

[54] O. Stier, M. Grundmann, and D. Bimberg, Electronic and optical properties of strained quantum dots modeled by 8-band k·p theory, Phys. Rev. B 59, 5688 (1999).

[55] C. Santori, D. Fattal, J. Vučković, G. S. Solomon, and Y. Yamamoto, Indistinguishable photons from a single-photon device, Nature (London) 419, 594 (2002).

[56] A. Kiraz, M. Atatüre, and A. Imamoğlu, Quantum-dot single-photon sources: Prospects for applications in linear optics quantum-information processing, Phys. Rev. A 69, 032305 (2004). 
[57] J. Johansen, S. Stobbe, I. S. Nikolaev, T. Lund-Hansen, P. T. Kristensen, J. M. Hvam, W. L. Vos, and P. Lodahl, Size dependence of the wave function of self-assembled InaAs quantum dots from time-resolved optical measurements, Phys. Rev. B 77, 073303 (2008).

[58] K. H. Madsen, S. Ates, J. Liu, A. Javadi, S. M. Albrecht, I. Yeo, S. Stobbe, and P. Lodahl, Efficient out-coupling of high-purity single photons from a coherent quantum dot in a photonic-crystal cavity, Phys. Rev. B 90, 155303 (2014).

[59] A. J. Bennett, J. P. Lee, D. J. P. Ellis, T. Meany, E. Murray, F. F. Floether, J. P. Griffths, I. Farrer, D. A. Ritchie, and A. J. Shields, Cavity-enhanced coherent light scattering from a quantum dot, Sci. Adv. 2, e1501256 (2016).

[60] F. Werschler, C. Hinz, F. Froning, P. Gumbsheimer, J. Haase, C. Negele, T. de Roo, S. Mecking, A. Leitenstorfer, D. V. Seletskiy, Coupling of excitons and discrete acoustic phonons in vibrationally isolated quantum emitters, Nano Lett. 16, 5861 (2016).
[61] Y. Masumoto and T. Takagahara, Semiconductor Quantum Dots: Physics, Spectroscopy and Applications (Springer Science \& Business Media, New York, 2013).

[62] R. Coles et al., Chirality of nanophotonic waveguide with embedded quantum emitter for unidirectional spin transfer, Nat. Commun. 7, 11183 (2016).

[63] M. Heiss et al., Self-assembled quantum dots in a nanowire system for quantum photonics, Nat. Mater. 12, 439 (2013).

[64] D. V. Anghel and T. Kühn, Quantization of the elastic modes in an isotropic plate, J. Phys. A 40, 10429 (2007).

[65] L. Midolo, S. N. Yoon, F. Pagliano, T. Xia, F. W. M. van Otten, M. Lermer, S. Höfling, and A. Fiore, Electromechanical tuning of vertically-coupled photonic crystal nanobeams, Opt. Express 20, 19255 (2012).

[66] A. Reigue et al., Probing Electron-Phonon Interaction through Two-Photon Interference in Resonantly Driven Quantum Dots, Phys. Rev. Lett. 118, 233602 (2017). 